\title{
DISCOVERY OF VERY HIGH ENERGY GAMMA RAYS FROM 1ES 1218+30.4
}

\section{J. Albert, ${ }^{1}$ E. Aliu, ${ }^{2}$ H. Anderhub,${ }^{3}$ P. Antoranz,${ }^{4}$ A. Armada,${ }^{2}$ M. Asensio, ${ }^{4}$ C. Baixeras, ${ }^{5}$ J. A. Barrio, ${ }^{4}$ M. Bartelt, ${ }^{6}$} H. Bartko, ${ }^{7}$ D. Bastieri, ${ }^{8}$ S. R. Bavikadi, ${ }^{9}$ W. Bednarek, ${ }^{10}$ K. Berger, ${ }^{1}$ C. Bigongiari, ${ }^{8}$ A. Biland, ${ }^{3}$ E. Bisesi, ${ }^{9}$ R. K. Bock, ${ }^{7}$ T. Bretz, ${ }^{1}$ I. Britvitch, ${ }^{3}$ M. Camara, ${ }^{4}$ A. Chilingarian, ${ }^{11}$ S. Ciprini, ${ }^{12}$ J. A. Coarasa, ${ }^{7}$ S. Commichau, ${ }^{3}$ J. L. Contreras, ${ }^{4}$ J. Cortina, ${ }^{2}$ V. Curtef, ${ }^{6}$ V. Danielyan,${ }^{11}$ F. Dazzi, ${ }^{8}$ A. De Angelis, ${ }^{9}$ R. De los Reyes, ${ }^{4}$ B. De Lotto, ${ }^{9}$ E. Domingo-Santamaría, ${ }^{2}$ D. Dorner, ${ }^{1}$ M. Doro,${ }^{8}$ M. Errando, ${ }^{2}$ M. Fagiolini, ${ }^{13}$ D. Ferenc, ${ }^{14}$ E. Fernández, ${ }^{2}$ R. Firpo, ${ }^{2}$ J. Flix, ${ }^{2}$ M. V. Fonseca, ${ }^{4}$ L. Font,${ }^{5}$ N. Galante, ${ }^{13}$ M. Garczarczyk, ${ }^{7}$ M. Gaug, ${ }^{2}$ M. Giller, ${ }^{10}$ F. Goebel, ${ }^{7}$ D. Hakobyan, ${ }^{11}$ M. Hayashida, ${ }^{7}$ T. Hengstebeck, ${ }^{15}$ D. Höhne, ${ }^{1}$ J. Hose, ${ }^{7}$ P. Jacon, ${ }^{10}$ O. Kalekin, ${ }^{15}$ D. Kranich, ${ }^{3,14}$ A. Laille, ${ }^{14}$ T. Lenisa, ${ }^{9}$ P. Liebing, ${ }^{7}$ E. Lindfors, ${ }^{12}$ F. Longo,${ }^{16}$ J. López, ${ }^{2}$ M. López, ${ }^{4}$ E. Lorenz, ${ }^{3,7}$ F. Lucarelli, ${ }^{4}$ P. Majumdar, ${ }^{7}$ G. Maneva, ${ }^{17}$ K. Mannheim, ${ }^{1}$ M. Mariotti,${ }^{8}$ M. Martínez, ${ }^{2}$ K. Mase, ${ }^{7}$ D. Mazin, ${ }^{7}$ M. Meucci, ${ }^{13}$ M. Meyer, ${ }^{1}$ J. M. Miranda, ${ }^{4}$ R. Mirzoyan, ${ }^{7}$ S. Mizobuchi,${ }^{7}$ A. Moralejo, ${ }^{7}$ K. Nilsson, ${ }^{12}$ E. Oña-Wilhelmi, ${ }^{2}$ R. Orduña,${ }^{5}$ N. Otte, ${ }^{7}$ I. Oya,${ }^{4}$ D. Paneque, ${ }^{7}$ R. Paoletti,${ }^{13}$ M. Pasanen,,${ }^{12}$ D. Pascoli,${ }^{8}$ F. Pauss,${ }^{3}$ N. Pavel,${ }^{15,18}$ R. Pegna, ${ }^{13}$ M. Persic, ${ }^{19}$ L. Peruzzo, ${ }^{8}$ A. Piccioli,${ }^{13}$ M. Poller,${ }^{1}$ E. Prandini, ${ }^{8}$ W. Rhode, ${ }^{6}$ J. Rico, ${ }^{2}$ B. Riegel,${ }^{1}$ M. Rissi, ${ }^{3}$ A. Robert, ${ }^{5}$ S. Rügamer, ${ }^{1}$ A. Saggion, ${ }^{8}$ A. Sánchez, ${ }^{5}$ P. Sartori, ${ }^{8}$ V. Scalzotto, ${ }^{8}$ R. Schmitt, ${ }^{1}$ T. Schweizer, ${ }^{15}$ M. Shayduk, ${ }^{15}$ K. Shinozaki ${ }^{7}$ S. N. Shore, ${ }^{20}$ N. Sidro, ${ }^{2}$ A. Sillanpää, ${ }^{12}$ D. Sobczyńska, ${ }^{10}$ A. Stamerra, ${ }^{13}$ L. S. Stark, ${ }^{3}$ L. Takalo, ${ }^{12}$ P. Temnikov, ${ }^{17}$ D. Tescaro, ${ }^{2}$ M. Teshima, ${ }^{7}$ N. Tonello, ${ }^{7}$ A. Torres, ${ }^{5}$ D. F. Torres,,${ }^{2,21}$ N. Turini, ${ }^{13}$ H. Vankov,${ }^{17}$ A. Vardanyan, ${ }^{11}$ V. Vitale, ${ }^{9}$ R. M. WAGNer, ${ }^{7}$ T. Wibig, ${ }^{10}$ W. Wittek, ${ }^{7}$ AND J. ZAPATERo ${ }^{5}$ Received 2006 February 22; accepted 2006 April 3; published 2006 April 24

\section{ABSTRACT}

The MAGIC collaboration has studied the high-frequency-peaked BL Lac object 1ES 1218+30.4, at a redshift $z=0.182$, using the MAGIC imaging air Cerenkov telescope located on the Canary Island of La Palma. A gamma-ray signal was observed with $6.4 \sigma$ significance. The differential energy spectrum for an energy threshold of $120 \mathrm{GeV}$ can be fitted by a simple power law, yielding $F_{E}(E)=(8.1 \pm 2.1) \times 10^{-7}[E /(250 \mathrm{GeV})]^{-3.0 \pm 0.4} \mathrm{TeV}^{-1}$ $\mathrm{m}^{-2} \mathrm{~s}^{-1}$. During the 6 days of observation in 2005 January, no time variability on timescales of days was found within the statistical errors. The observed integral flux above $350 \mathrm{GeV}$ is nearly a factor of 2 below the upper limit reported by the Whipple collaboration in 2003.

Subject headings: BL Lacertae objects: individual (1ES 1218+30.4) — gamma rays: observations

\section{INTRODUCTION}

Since the detection of blazars as sources of high-energy gamma rays by the Energetic Gamma-Ray Experiment Telescope (EGRET) on board the Compton Gamma Ray Observatory, the search for the very high energy (VHE) counterparts of these sources has been a major goal for ground-based gamma-ray astronomy. The first blazar detected at VHE energies was Mrk 421 (Punch et al. 1992). Subsequent to the discovery of Mrk 421, only a small number of extragalactic

\footnotetext{
${ }^{1}$ Lehrstuhl für Astronomie, Universität Würzburg, Am Hubland, D-97074 Würzburg, Germany; correspondence should be addressed to M. Meyer, meyer@astro.uni-wuerzburg.de.

${ }^{2}$ Institut de Física d'Altes Energies, Universitat Autònoma de Barcelona, E-08193 Bellaterra, Spain.

${ }^{3}$ Institute for Particle Physics, ETH Hönggerberg, CH-8093 Zurich, Switzerland.

${ }^{4}$ Grupo de Altas Energías, Departamento de Física Atómica, Molecular y Nuclear, Universidad Complutense de Madrid, Avenida Complutense s/n, E-28040 Madrid, Spain.

${ }^{5}$ Grup de Física de les Radiacions, Departament de Física, Universitat Autònoma de Barcelona, E-08193 Bellaterra, Spain.

${ }^{6}$ Fachberich Physik, Universität Dortmund, D-44227 Dortmund, Germany.

${ }^{7}$ Max-Planck-Institut für Physik, Föhringer Ring 6, D-80805 Munich, Germany.

${ }^{8}$ Dipartimento di Fisica, Università di Padova, and INFN Padova, via Marzolo 8, I-35131 Padua, Italy.

${ }^{9}$ Dipartimento di Fisica, Università di Udine, and INFN Trieste, via delle Scienze 208, I-33100 Udine, Italy.

${ }^{10}$ Instytut Fizyki, Uniwersytet Łódzki, ulica Pomorska 149/153, PL-90236 Lodz, Poland.
}

VHE sources were found, although the sensitivity of groundbased telescopes is superior in their energy range to that of EGRET. The total number of VHE blazars reported in the literature as of 2006 February amounts to 11, while the number of high-confidence blazars in the third EGRET catalog is 66 (Hartman et al. 1999).

Stecker et al. (1992) pointed out that the attenuation of gamma rays due to photon-photon interactions with low-energy photons from the extragalactic background radiation was a likely explanation of this deficit. In fact, the redshifts of blazars

\footnotetext{
${ }^{11}$ Cosmic Ray Division, Yerevan Physics Institute, 2 Alikhanyan Yeghbayrneri, 375036 Yerevan, Armenia.

${ }^{12}$ Tuorla Observatory, Väisälänte 20, FI-21500 Piikkiö, Finland.

${ }^{13}$ Dipartimento di Fisica, Università di Siena, and INFN Pisa, via Roma 56, I-53100 Siena, Italy.

${ }^{14}$ Department of Physics, University of California, Davis, 1 Shields Avenue, Davis, CA 95616.

${ }^{15}$ Institut für Physik, Humboldt-Universität zu Berlin, Newtonstrasse 15, D-12489 Berlin, Germany

${ }^{16}$ Dipartimento di Fisica, Università di Trieste, and INFN Trieste, Padriciano 99, I-34012 Trieste, Italy.

${ }^{17}$ Institut za Yadreni Izsledvaniya i Yadena Energetika, Bulevard Tsarigradsko shose, BG-1784 Sofia, Bulgaria.

${ }^{18}$ Deceased.

${ }^{19}$ Osservatorio Astronomico di Trieste, INAF, and INFN Trieste, via Tiepolo 11, I-34100 Trieste, Italy.

${ }^{20}$ Dipartimento di Fisica, Università di Pisa, and INFN Pisa, Largo Pontecorvo 3, I-56126 Pisa, Italy.

${ }^{21}$ Institut de Ciències de l'Espai, Universitat Autònoma de Barcelona, E-08193 Bellaterra, Spain
} 
detected so far above $100 \mathrm{GeV}$ are at rather low values as expected from predictions of the correlation between the gamma-ray attenuation and the redshift of the source, known as the Fazio-Stecker relation (Fazio \& Stecker 1970; Kneiske et al. 2004). These primary gamma rays, absorbed by pair production, turn into secondary gamma rays contributing to the extragalactic background light (Aharonian et al. 1994).

The VHE gamma-ray-emitting blazars known so far all belong to the class of high-frequency-peaked BL Lacertae objects (HBLs; Padovani \& Giommi 1995), a subclass of blazars characterized by a low luminosity compared with quasars and a synchrotron peak in the X-ray band. Their spectral energy distribution (SED) is characterized by a second peak at very high gamma-ray energies. In synchrotron self-Compton (SSC) models, it is assumed that the observed gamma-ray peak is due to inverse Compton emission from the accelerated electrons upscattering previously produced synchrotron photons to high energies (Maraschi et al. 1992). In hadronic models, secondary electrons and positrons, arising from photomeson production, initiate electromagnetic cascades (Mannheim 1993; Mücke \& Protheroe 2001). A compilation of blazars with known X-ray spectra allowing their classification as HBLs is given by Donato et al. (2001).

During the first year of operation of the Major Atmospheric Gamma Imaging Cherenkov (MAGIC) Telescope, a sample of $\mathrm{X}$-ray-bright $\left(F_{1 \mathrm{kev}}>2 \mu \mathrm{Jy}\right)$, northern HBLs at moderate redshifts $(z<0.3)$, including 1ES $1218+30.4$, which is located at a redshift $z=0.182$ (Véron-Cetty \& Véron 2003), have been observed. Predictions based on models invoking an SSC (Costamante \& Ghisellini 2002) or hadronic (Mannheim et al. 1996) origin of the gamma rays and the shape of the time-averaged SED show that these HBLs should be detectable by imaging air Cerenkov telescopes (IACTs), although most of them are too faint in the EGRET energy range to have been detected during its all-sky survey.

For 1ES $1218+30.4$, the EGRET upper limit is $F(>100$ $\mathrm{MeV}) \approx 10^{-11} \operatorname{ergs~} \mathrm{cm}^{-2} \mathrm{~s}^{-1}$. Several observations with the Whipple telescope between 1995 and 2000 resulted in an upper flux limit of $F(>350 \mathrm{GeV})=8.3 \times 10^{-8}$ photons $\mathrm{m}^{-2} \mathrm{~s}^{-1}$ (corresponding to $\sim 8 \%$ of the Crab Nebula flux; Horan et al. 2004). The source was also observed by the HEGRA experiment between 1996 and 2002. An upper flux limit above $840 \mathrm{GeV}$ of $2.67 \times 10^{-8}$ photons $\mathrm{m}^{-2} \mathrm{~s}^{-1}$ (or $12 \%$ of the Crab Nebula flux) is reported by Aharonian et al. (2004).

In this Letter, we report the first detection of VHE gamma rays $(>100 \mathrm{GeV})$ from the direction of $1 \mathrm{ES} 1218+30.4$. In $\S 2$, we briefly discuss the observations and the data set, while we describe in $\S 3$ the data analysis method and the results. In $\S 4$, we discuss the results in the context of the SED of 1ES $1218+30.4$ across a broad energy range.

\section{OBSERVATIONS}

The MAGIC Telescope is a single-dish IACT, located on the Canary Island of La Palma (N28.8, W17.8, $2200 \mathrm{~m}$ above sea level). A $17 \mathrm{~m}$ diameter tessellated parabolic mirror with a total surface of $234 \mathrm{~m}^{2}$, mounted on a lightweight space frame made from carbon-fiber-reinforced plastic tubes, focuses Cerenkov light from air showers, initiated by gamma rays or charged cosmic rays, onto a 576 pixel photomultiplier (PMT) camera with a field of view of 3.5 . The analog signals are transported by means of optical fibers to the trigger electronics, and each channel is read out by a $300 \mathrm{MHz}$ flash analog-to-digital con- verter. Further details on the telescope can be found in Baixeras et al. (2004) and Cortina et al. (2005).

MAGIC observed 1ES $1218+30.4$ from 2005 January 9 to 15 at zenith angles ZA between 1.5 and $13^{\circ}$ during six moonless nights, for a total observation time of $8.2 \mathrm{hr}$. To determine the background, so-called OFF data were taken in addition, by pointing the telescope to a nearby sky region where no gammaray source was expected. The OFF data cover the same ZA range, with a similar night-sky background light intensity. For the analysis, 6.5 hours' OFF data, taken between January 9 and January 11, were used, which match the observation conditions and detector performance of the $\mathrm{ON}$ data. At the same time, the KVA telescope ${ }^{22}$ on La Palma observed 1ES $1218+30.4$ in the optical range.

The signal was discovered with our automatic analysis (Dorner et al. 2005). The final analysis is described below.

\section{DATA ANALYSIS AND RESULTS}

The data were processed using the MAGIC Analysis and Reconstruction Software (MARS; Bretz 2005). A description of the different analysis steps can be found in Gaug et al. (2005) (including the calibration) and Bretz et al. (2005). The moments up to third order of the light distribution are used to characterize each event by a set of image parameters (Hillas 1985). For background suppression, a SIZE-dependent parabolic cut in WIDTH $\times$ LENGTH is applied (Riegel et al. 2005).

To reconstruct the origin of the shower in the camera plane, the DISP method is employed (Lessard et al. 2001) to estimate the distance between the center of gravity of the shower and its origin. The third moment determines the direction of the shower's development. The constant coefficient $\xi$ from the parameterization of DISP in the original approach is replaced by $\xi_{0}+\xi_{1} \times(\text { LEAKAGE })^{\xi_{2}}$, LEAKAGE being the fraction of light contained in the outermost camera pixels. Thereby the truncation of shower images at the camera border is taken into account. These coefficients were determined using simulated data.

Simulated gamma showers (Monte Carlo) were produced with CORSIKA, version 6.023 (Heck et al. 1998; ${ }^{23}$ Majumdar et al. 2005) for $\mathrm{ZA}$ below $20^{\circ}$ and energies between $10 \mathrm{GeV}$ and $30 \mathrm{TeV}$, following a power law with a spectral index -2.6 . The cut coefficients for the background suppression were optimized using data for the Crab Nebula, taken at similar ZA in a time slot of several weeks around the observation.

Figure 1 shows the background-subtracted distribution of reconstructed shower origins, centered at the position of 1ES $1218+30.4$. Each shower origin is folded with a two-dimensional Gaussian with a standard deviation of 0.06 , corresponding to half the $\sigma$ of the point-spread function expected for a pointlike gamma-ray source as seen by MAGIC. The center of gravity of the observed excess coincides within the systematic pointing uncertainty of 0.04 with the nominal source position.

Figure 2 shows the distribution of the squared angular distance, $\theta^{2}$, between the reconstructed and the nominal source positions for both the $\mathrm{ON}$ and the OFF data. The background distribution is not flat, as a consequence of the limited trigger area, which means that the highest acceptance of the detector is in the center and decreases outward. Because of the differing observation times, the OFF data were scaled to match the ON data in the region $0.14<[\theta(\mathrm{deg})]^{2}<0.64$, where no bias from

\footnotetext{
${ }^{22}$ See http://tur3.tur.iac.es.

${ }^{23} \mathrm{See}$ http://wwwik.fzk.de/corsika/physics_description/corsika_phys.html.
} 


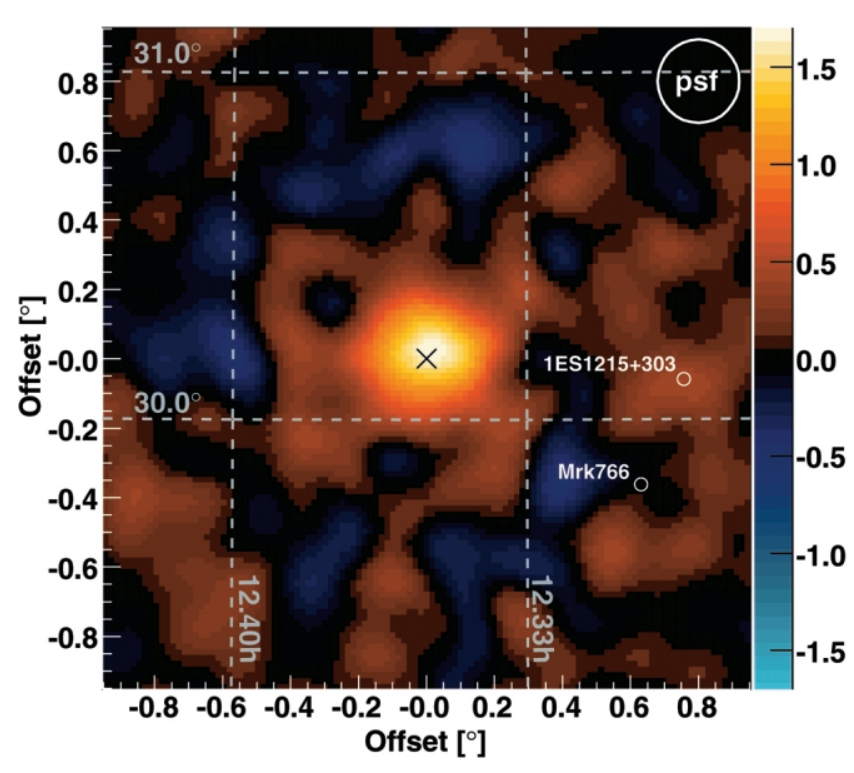

FIG. 1.-Sky map of the region around the position of 1ES $1218+30.4$ (cross) for gamma-ray events, with an energy threshold of $\sim 140 \mathrm{GeV}$. The scale is in units of 10 events per square arcminute.

the source is expected. The observed excess of 560 events has a statistical significance of 6.4 standard deviations (according to Li \& Ma 1983). The energy threshold, defined as the peak energy of simulated gamma rays with a differential spectrum proportional to $E^{-3.0}$ surviving all cuts, is $\sim 140 \mathrm{GeV}$.

For the energy estimation, the random-forests regression method was applied (Breiman 2001), trained with the aforementioned Monte Carlo (MC) sample, yielding an average energy resolution of 24\%. Figure 3 and Table 1 show the reconstructed energy spectrum. To avoid systematic effects of cut efficiencies to the MC sample, the acceptance window for gamma rays was enlarged, yielding an energy threshold of $\sim 120 \mathrm{GeV}$. Spillover effects to neighboring bins were corrected by the number ratio between the energy distribution of an MC sample compared with the distribution of estimated energy. A power law was fitted to the measured spectral points, yielding

$$
F_{E}(E)=(8.1 \pm 2.1) \times 10^{-7}\left(\frac{E}{250 \mathrm{GeV}}\right)^{-3.0 \pm 0.4} \mathrm{~m}^{-2} \mathrm{~s}^{-1} \mathrm{TeV}^{-1}
$$

$\left(\chi^{2}=1.1\right.$ with 4 degrees of freedom [dof]).

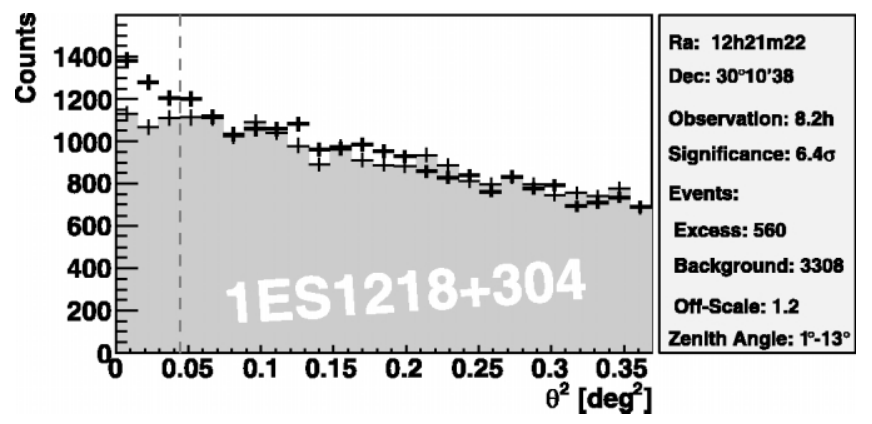

FIG. 2.-Distribution of $\theta^{2}$ for ON and OFF data (gray). The signal region is marked by the dashed line.

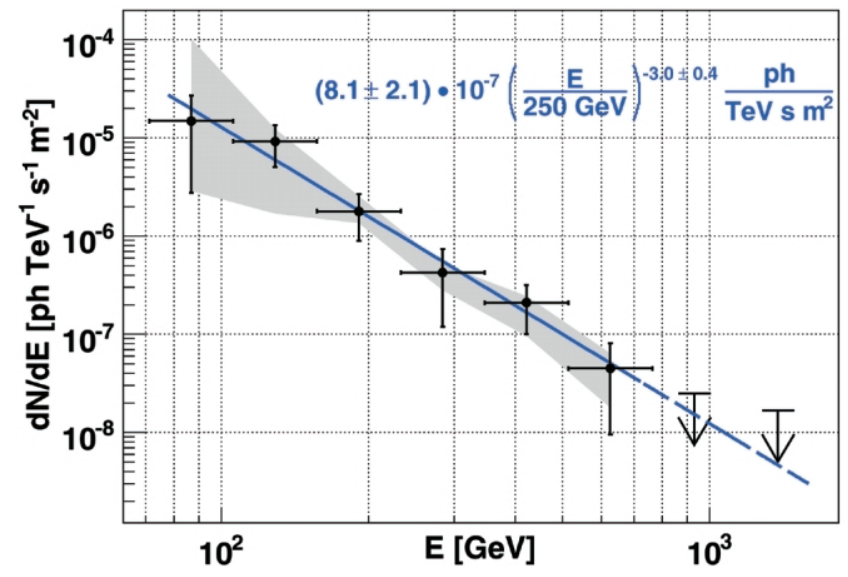

FIG. 3.-Differential energy spectrum of 1ES $1218+30.4$. The upper limits correspond to the $90 \%$ confidence level.

The error bars $(1 \sigma)$ show the statistical uncertainty only. The systematic error resulting from the analysis was investigated by changing cut coefficients and the initial MC spectrum (Fig. 3, gray region). The total systematic error for the spectral slope is estimated to be ${ }_{-0.4}^{+0.7}$. Additional systematics for the flux level are estimated to be on the order of $\sim 40 \%$. The main contributions are uncertainties in the atmospheric conditions, the mirror reflectivity, and the effective quantum efficiency of the PMTs.

The data were also analyzed with two other, independent analysis techniques, using either dynamical supercuts (Kranich 1997) or the random-forests method for background suppression, as used in previous MAGIC observations (e.g., Albert et al. 2006). Within the statistical errors, the same significance, flux, and differential spectrum were obtained.

For the analysis of time variability, the sample was divided into six subsamples, one for every night of observation, with $\sim 85$ minutes' observation time each. Figure 4 shows the integral flux above $100 \mathrm{GeV}$ for each night. Within the statistical errors, we obtain a constant flux $\left(\chi^{2} / \mathrm{dof}=2.4 / 5\right)$ of $F(>100 \mathrm{GeV})=(8.7 \pm 1.4) \times 10^{-7}$ photons $\mathrm{m}^{-2} \mathrm{~s}^{-1}$. The systematic error is on the order of $\sim 40 \%$, as discussed above. The integral flux is $\sim 30 \%$ higher than one would expect from integration of equation (1). This can be understood by taking into account the fact that the flux at $130 \mathrm{GeV}$ (the peak of the event distribution) lies $\sim 35 \%$ above the fit value.

\section{DISCUSSION AND CONCLUSIONS}

The discovery of VHE gamma-ray emission from 1ES $1218+30.4$ provides further evidence that HBLs generally ex-

TABLE 1

DifFerential Flux of 1ES 1218+30.4 AND Number of Measured Excess Events

\begin{tabular}{ccr}
\hline \hline $\begin{array}{c}E \\
(\mathrm{GeV})\end{array}$ & \multicolumn{1}{c}{$\begin{array}{c}F_{E}(E) \\
\left(\mathrm{m}^{-2} \mathrm{~s}^{-1} \mathrm{TeV}^{-1}\right)\end{array}$} & \multicolumn{1}{c}{$N_{\mathrm{ex}}$} \\
\hline $87 \ldots \ldots$ & $\left(1.5 \pm 1.2_{-1.2}^{+8.7}\right) \times 10^{-5}$ & $27 \pm 21$ \\
$130 \ldots \ldots$ & $\left(9.2 \pm 4.2_{-7.5}^{+3.1}\right) \times 10^{-6}$ & $163 \pm 73$ \\
$190 \ldots \ldots$ & $\left(1.8 \pm 0.9_{-0.4}^{+0.8}\right) \times 10^{-6}$ & $146 \pm 71$ \\
$280 \ldots \ldots$ & $\left(4.3 \pm 3.1_{-1.4}^{+0.9}\right) \times 10^{-7}$ & $64 \pm 46$ \\
$420 \ldots \ldots$ & $\left(2.1 \pm 1.1_{-1.1}^{+0.4}\right) \times 10^{-7}$ & $61 \pm 30$ \\
$630 \ldots \ldots$ & $\left(4.5 \pm 3.5_{-2.7}^{+1.9}\right) \times 10^{-8}$ & $22 \pm 17$ \\
\hline
\end{tabular}

Note.-Measured values are given together with the (symmetric) statistical error. For the flux, the systematic error is also listed (see text for details). 


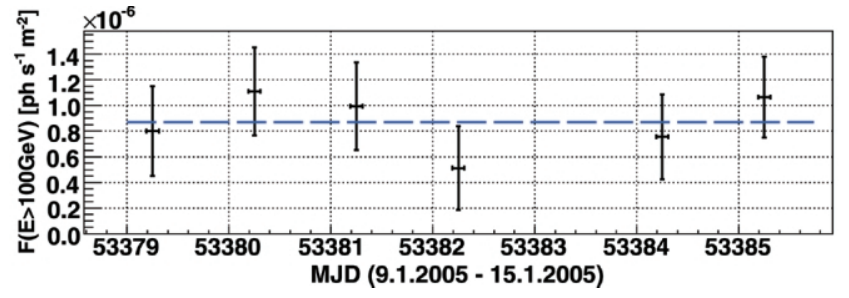

FIG. 4.-Integral flux ( $>100 \mathrm{GeV})$ of 1ES $1218+30.4$ for each subsample. The error bars $(1 \sigma)$ show the statistical uncertainty only.

hibit gamma-ray emission at a luminosity comparable to the $\mathrm{X}$-ray luminosity. Archival X-ray data show that the X-ray flux at $1 \mathrm{keV}$ varies in the range $(0.8-4.5) \times 10^{-11} \mathrm{ergs} \mathrm{cm}^{-2} \mathrm{~s}^{-1}$ (White et al. 2000), which is below the sensitivity of the AllSky Monitor on board the RXTE X-ray satellite. The observed gamma-ray flux at $100 \mathrm{GeV}$ is $(1.9 \pm 1.1) \times 10^{-11} \mathrm{ergs} \mathrm{cm}^{-2}$ $\mathrm{s}^{-1}$, at the same level as published X-ray fluxes. Contemporaneous observations from Swift in 2005 October showed a flux comparable to that measured by BeppoSAX in 1999 (P. Giommi 2006 , in preparation), which is also within the range of the value measured by ROSAT.

There are no indications from simultaneous optical observations (KVA) that a flare occurred during the time of the gamma-ray observations, nor does the gamma-ray light curve show signs of significant variability. The spectrum is consistent with upper limits at higher energies determined in the past.
Taking the upper limit of EGRET into account, the gammaray peak of the SED is constrained to lie in the $1-100 \mathrm{GeV}$ regime, unless attenuation of the gamma rays by pair production in the metagalactic radiation field masks an emitted bump in the spectrum at higher energies. Detailed modeling of the attenuation thus becomes the task to unfold the emitted spectrum from the observed one. Sizable attenuation is expected from current models of the evolving extragalactic (i.e., metagalactic) background light (Hauser \& Dwek 2001; Kneiske et al. 2004).

At hard X-ray energies, SSC models predict a sharp rollover in the SED, in contrast to the predictions of hadronic models involving electromagnetic cascades. Observations in the hard X-ray domain, for example, using Swift or Suzaku (Astro-E2), and in the intermediate gamma-ray regime with the GammaRay Large Area Space Telescope, will be crucial to provide information about the missing link between the synchrotron peak and the gamma-ray peak.

We would like to thank the Instituto de Astrofísica de Canarias for the excellent working conditions at the Observatorio del Roque de los Muchachos in La Palma. The support of the German Bundesministerium für Bildung und Forschung and Max-Planck-Gesellschaft, the Italian Istituto Nazionale de Fisica Nucleare, and the Spanish Comisión Interministerial de Ciencias y Tecnología is gratefully acknowledged. This work was also supported by ETH Research Grant TH 34/04 3 and grant 1P03D01028 from the Polish Ministerstwo Nauki i Informatyzacji.

\section{REFERENCES}

Aharonian, F., et al. 2004, A\&A, 421, 529

Aharonian, F. A., Coppi, P. S., \& Völk, H. J. 1994, ApJ, 423, L5

Albert, J., et al. 2006, ApJ, 637, L41

Baixeras, C., et al. 2004, Nucl. Instrum. Meth. Phys. Res. A, 518, 188

Breiman, L. 2001, Machine Learning, 45, 5

Bretz, T. 2005, in AIP Conf. Proc. 745, High Energy Gamma-Ray Astronomy, ed. F. A. Aharonian, H. J. Völk, \& D. Horns (New York: AIP), 730

Bretz, T., et al. 2005, Proc. 29th Int. Cosmic Ray Conf. (Pune), 4, 315

Cortina, J., et al. 2005, Proc. 29th Int. Cosmic Ray Conf. (Pune), 5, 359

Costamante, L., \& Ghisellini, G. 2002, A\&A, 384, 56

Donato, D., Ghisellini, G., Tagliaferri, G., \& Fossati, G. 2001, A\&A, 375, 739

Dorner, D., Berger, K., Bretz, T., \& Gaug, M. 2005, Proc. 29th Int. Cosmic Ray Conf. (Pune), 5, 175

Fazio, G. G., \& Stecker, F. W. 1970, Nature, 226, 135

Gaug, M., Bartko, H., Cortina, J., \& Rico, J. 2005, Proc. 29th Int. Cosmic Ray Conf. (Pune), 5, 375

Hartman, R. C., et al. 1999, ApJS, 123, 79

Hauser, M. G., \& Dwek, E. 2001, ARA\&A, 39, 249

Heck, D., Knapp, J., Capdevielle, J. N., Schatz, G., \& Thouw, T. 1998, CORSIKA: A Monte Carlo Code to Simulate Extensive Air Showers (Rep. FZKA 6019) (Karlsruhe: Forschungszentrum Karlsruhe)

Hillas, A. M. 1985, Proc. 19th Int. Cosmic Ray Conf. (La Jolla), 3, 445
Horan, D., et al. 2004, ApJ, 603, 51

Kneiske, T. M., Bretz, T., Mannheim, K., \& Hartmann, D. H. 2004, A\&A, 413, 807

Kranich, D. 1997, Ph.D. thesis, Tech. Univ. München

Lessard, R. W., Buckley J. H., Connaughton, V., \& Le Bohec, S. 2001, Astropart. Phys., 15, 1

Li, T., \& Ma, Y. 1983, ApJ, 272, 317

Majumdar, P., Moralejo, A., Bigongiari, C., Blanch, O., \& Sobczyńska, D. 2005, Proc. 29th Int. Cosmic Ray Conf. (Pune), 5, 203

Mannheim, K. 1993, A\&A, 269, 67

Mannheim, K., Westerhoff, S., Meyer, H. \& Fink, H.-H. 1996, A\&A, 315, 77

Maraschi, L., Ghisellini, G., \& Celotti, A. 1992, ApJ, 397, L5

Mücke, A., \& Protheroe, R. J. 2001, Astropart. Phys., 15, 121

Padovani, P., \& Giommi, P. 1995, ApJ, 444, 567

Punch, M., et al. 1992, Nature, 358, 477

Riegel, B., Bretz, T., Dorner, D., Kerger, K., \& Höhne, D. 2005, Proc. 29th Int. Cosmic Ray Conf. (Pune), 5, 215

Stecker, F. W., De Jager, O. C., \& Salamon, M. H. 1992, ApJ, 390, 49

Véron-Cetty, M.-P. \& Véron, P. 2003, A\&A, 412, 399

White, N. E., Giommi, P., \& Angelini, L. 2000, The WGA Catalogue of ROSAT Point Sources (Greenbelt, MD: GSFC) 